\title{
Canadian guidelines for treatment of non-muscle invasive bladder cancer: a focus on intravesical therapy
}

\author{
Wassim Kassouf, MD, FRCSC; ${ }^{*}$ Ashish M. Kamat, MD, FRCSC; ${ }^{\dagger}$ Alexander Zlotta, MD, FRCSC,; Bernard H. \\ Bochner, MD, FRCSC; $;$ Ronald Moore, MD, FRCSC; ${ }_{;}^{*}$ Alan So, MD, FRCSC; ${ }^{* *}$ Jonathan Izawa, MD, FRCSC; ;t \\ Ricardo A. Rendon, MD, FRCSC, ${ }^{\neq \neq}$Louis Lacombe, MD, FRCSC, ${ }^{\text {ss }}$ Armen G. Aprikian, MD, FRCSC ${ }^{*}$
}

Can Urol Assoc J 2010;4(3):168-73

\section{Introduction}

In 2008, bladder cancer was estimated to be the fourth most common male cancer accounting for $6 \%$ of all cancers and the eighth highest cancer-related mortality rate in Canadian men. The most common type is urothelial carcinoma (greater than 90\%), followed by adenocarcinoma, squamous cell and small cell carcinoma. Risk factors that have been associated with bladder cancer include smoking, chronic inflammatory changes in the bladder (due to persistent bladder stones, recurrent urinary tract infections, indwelling catheters or schistosomiasis) and chemotherapeutic exposure, such as cyclophosphamide. ${ }^{1-6}$ Other risk factors include pelvic irradiation, occupational exposure to chemicals from the aromatic amines family and chronic phenacetin use..$^{7-10}$ Nonmuscle invasive bladder cancer (NMIBC) accounts for about $75 \%$ to $80 \%$ of all bladder cancer cases; ${ }^{11}$ Ta accounts for most NMIBC $(60 \%)$, whereas $\mathrm{T} 1$ and Tis (carcinoma in situ [CIS]) account for $30 \%$ and $10 \%$, respectively. The actual prevalence of NMIBC is 10 times its incidence and creates a major economic burden on health-care systems. ${ }^{12}$ As measured on the basis of cumulative per patient cost from diagnosis until death, bladder cancer is the most expensive human cancer to treat. ${ }^{13}$ The management of NMIBC has significantly changed over the last decade. Canadian guidelines on the treatment of NMIBC are lacking. This manuscript provides a Canadian consensus on the management of NMIBC, with an emphasis on intravesical therapy, a comprehensive review of the literature and a review of the guidelines from the European Association of Urology, the American Urological Association and the National Comprehensive Cancer Network. ${ }^{14-16}$

\section{Prognostic factors for recurrence and progression of NMIBC}

The overall rate of recurrence for NMIBC is $60 \%$ to $70 \%$, and the overall rate of progression to a higher stage or grade is $20 \%$ to $30 \%{ }^{17,18}$ The two most important prognostic fac- tors in NMIBC are stage and grade. Ta tumours (which are usually low grade) rarely progress to a higher stage, but tend to recur frequently. On the other hand, T1 tumours (which are usually high grade) have the potential to be more aggressive, with higher rates of progression to muscle invasion and even metastasis. Heney and colleagues have shown that the risk of disease progression to muscle invasion is strongly associated with tumour grade. ${ }^{11}$ The risk of progression for Ta tumour was $2 \%, 11 \%$ and $45 \%$ for grades 1,2 , and 3 respectively. When stratified by stage, tumour grade continues to correlates with progression and mortality as stage increases. Most subsequent studies also suggested that grade is a better prognostic indicator of progression and mortality than of recurrence. ${ }^{19-22}$ However, recurrence is still a significant problem in the management of superficial bladder cancer. As many as $60 \%$ to $90 \%$ of NMIBC will recur if treated by transurethral resection (TUR) alone. ${ }^{23}$ Using 6 clinicopathologic parameters (grade, stage, tumour size, prior recurrence rate, presence of concomitant CIS and number of tumours), the probability of recurrence and progression of NMIBC can be calculated with risk tables provided by the European Organization for Research and Treatment of Cancer (EORTC), that were developed and based on individual patient data from 2596 patients diagnosed with Ta/T1 tumours who were randomized in 7 EORTC trials (www.eortc.be/tools/bladdercalculator). In general, patients with NMIBC can be stratified into low-risk disease (solitary, low-grade Ta lesion, $<3 \mathrm{~cm}$ ), intermediate $(>3 \mathrm{~cm}$, multiple, or multi-recurrent low-intermediate grade tumours), and high-risk (high-grade Ta, T1 tumours or CIS disease).

\section{TURBT}

Transurethral resection of bladder tumour (TURBT) is the first and gold standard treatment option for NMIBC. Complete resection of the tumour should be performed including all areas of suspected CIS and abnormal areas in the prostatic urethra and bladder neck. If extensive CIS is present, mapping biopsies should be obtained, and all visible papillary lesions resected. Transurethral resection of bladder tumour should include detrusor muscle (muscularis propria) in the 
specimen in an attempt to rule out $\mathrm{T} 2$ disease and minimize the risk of under-staging (Grade A recommendation). It not only eradicates all visible tumours, it also provides tissue for pathological analysis and determination of histological type and grade together with detecting the presence, depth and type of invasion. The quality of the initial TURBT specimen is extremely important. ${ }^{24}$ Several studies demonstrate that the quality of the TURBT can be improved using fluorescent cystoscopy. Integration of fluorescent cystoscopy during TURBT has been shown to reduce recurrence rates (Level of evidence 2). ${ }^{25,26}$ However, false positives with fluorescent cystoscopy can be increasingly induced by inflammation, recent TUR or intravesical instillations. Although fluorescent cystoscopy reduces recurrence rates, it is not certain if the long-term recurrence-free survival is affected. Furthermore, it is unclear whether fluorescent cystoscopy improves progression-free and overall survival in patients with NMIBC. At the present time, the use of combined fluorescent and white light TUR is optional (Grade C recommendation).

\section{Second TURBT}

Restaging TUR provides more tissue for pathologic examination and better staging as well as insight into the biology of the disease (Level of evidence 2). In patients with T1G3/T1HG, re-TUR upstaged tumours to T2 disease in $49 \%$ of patients if muscularis propria was not present in the specimen compared to $14 \%$ upstage if the initial TUR showed benign muscularis propria. ${ }^{27}$ Restaging TUR is also associated with better local control of tumour (Level of evidence 3 ). Herr previously reported that $75 \%$ of patients who underwent repeat TURBT in 2 to 6 weeks after initial resection have residual tumour. Forty-four percent of them were found to have $\mathrm{T} 1$ or muscle invasive tumour. Furthermore, $17 \%$ of patients who had TURBT of primary cancers actually have histological evidence of cancer at previous resection site despite a normal cystoscopic examination. ${ }^{28}$ After 5 years of follow-up in 124 patients, Grimm and colleagues found that $63 \%$ of those who underwent a repeat TURBT had tumour-free bladders compared with $40 \%$ of those who did not. ${ }^{29}$ Similarly, a recent study demonstrated that a restaging TUR improved initial response to intravesical immunotherapy. ${ }^{24}$ We recommend that a second TUR should always be performed 4 to 6 weeks after the initial resection when the initial TUR is incomplete or a $\mathrm{T} 1$ tumour is detected in the absence of muscularis propria in the specimen (Grade A recommendation). A second TUR is also recommended for any high-grade tumours or T1 tumours with benign muscularis propria in the specimen (Grade C recommendation). Collectively, removing all residual tumours in a second therapeutic TURBT allows for more accurate staging, improves patient selection (and thus response) to BCG therapy, reduces the frequency of recurrence and potentially delays tumour progression (Level of evidence 2). ${ }^{29}$

\section{Follow-up}

All patients are recommended to undergo a cystoscopy at 3 months following TURBT as cystoscopic findings at 3 months have been shown to be a prognostic factor of recurrence and progression of disease (Grade A recommendation). ${ }^{30}$ Prospective studies to better refine the surveillance schedule are sorely needed. Although there is no consensus for surveillance strategies, our general recommendation is to perform a follow-up cystoscopy with urine cytology (or other urine marker) every 3 to 4 months for 2 years, then every 6 months for the second 2 years, then yearly thereafter (Grade B recommendation). Patients with a primary, solitary, low-grade Ta tumour may have less frequent cystoscopic examination ( 3 and 9 months, then annually thereafter) (Level of evidence 3). Any recurrence resets the clock in the follow-up schedule. Annual upper tract imaging is recommended for patients with intermediate- and high-risk NMIBC (Grade C recommendation).

\section{Intravesical therapy}

Intravesical therapy can be either chemotherapy or immunotherapy and is either therapeutic (treatment of CIS or residual non-visible tumour), prophylactic (prevention of recurrence and progression of disease) or adjuvant in the immediate postoperative setting.

\section{Chemotherapy}

\section{Single immediate postoperative instillation}

A high proportion of patients with NMIBC will develop recurrences with a significant number recurring 3 months following TURBT. Incomplete TUR or tumour cell implantation post-TUR has been implicated to be responsible for the high recurrences at 3 months. As such, several studies have evaluated the role of a single postoperative intravesical instillation of chemotherapy. The commonly used intravesical chemotherapeutic agents are doxorubicin, epirubicin and mitomycin C (MMC).

Sylvester and colleagues performed a meta-analysis of 7 randomized trials $(n=1476)$ on the outcome of TUR alone versus TUR plus 1 immediate postoperative instillation of intravesical chemotherapy. ${ }^{31}$ Over a median follow-up of 3.4 years, patients who received one immediate instillation had a recurrence rate of $37 \%$ compared with $48 \%$ of patients who had TUR alone. The benefit was more pronounced for those with single, low-grade papillary tumour compared with patients with multiple tumours. The efficacy of the immedi- 


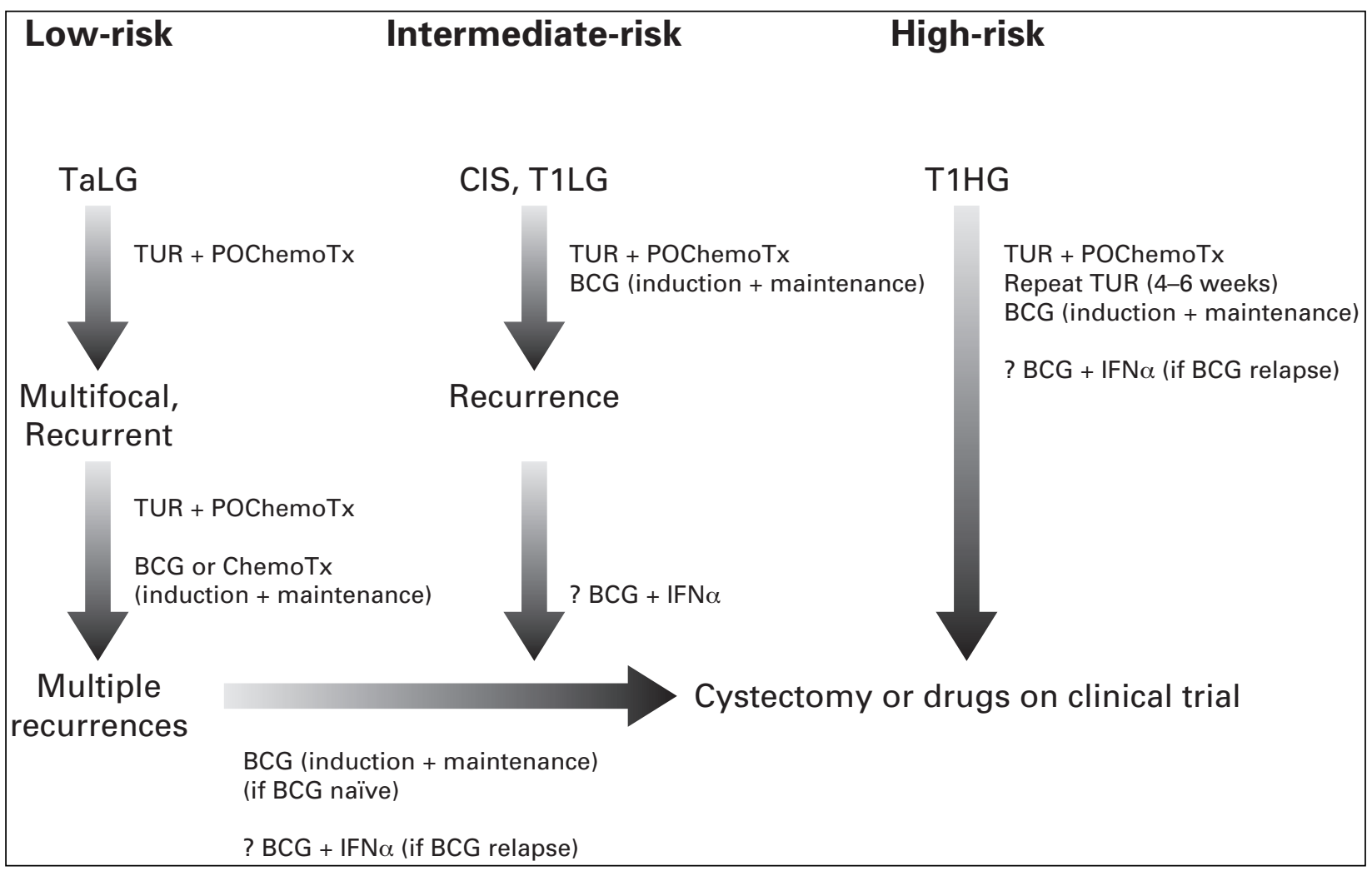

Fig. 1. Non-muscle invasive urinary carcinoma of the bladder. TUR = transurethral resection; $P O C h e m o T x=$ chemotherapy, orally; $B C G=$ bacillus Calmette-Guerin; IFN-alpha= interferon-alpha; TaLG = Ta low-grade; CIS = carcinoma in situ; T1LG = T1 low-grade; T1HG = T1 high-grade .

ate postoperative instillation is within 6 hours from the time of TUR and significantly decreases if given beyond 24 hours (Level of evidence 2). ${ }^{32}$ Immediate postoperative instillation of chemotherapeutic agent is recommended for all patients with NMIBC after TURBT (Grade B recommendation); for patients who are planned to be treated with bacillus CalmetteGuerin (BCG), the use of an immediate postoperative instillation of chemotherapy is optional as its benefit in this situation is less clear (Grade D recommendation). Overall, long-term recurrence reduction is similar between the different chemotherapeutic agents, being in the range of about $15 \%$. The most commonly used intravesical chemotherapeutic agent in Canada is MMC. Since the molecule has a high molecular weight, absorption and myelosuppression due to MMC are uncommon. Patients with suspected bladder perforation should not receive an immediate instillation as severe complications have been reported in this setting. ${ }^{33-35}$ Efficacy of MMC are dependent on the concentration at which the drug is administered. The dose commonly used is $40 \mathrm{mg}$ in $40 \mathrm{~mL}$ of saline or water (with some using $40 \mathrm{mg}$ in $20 \mathrm{~mL}$ ). Recently, $\mathrm{Au}$ and colleagues published a phase III, randomized trial that showed superiority and prolonged median time to recurrence with an "optimized" MMC administration which consist of a period of dehydration (no fluids for 8 hours prior to treatment), urinary alkalinization, confirmation of complete bladder drainage prior to instillation and a higher MMC concentration (40 mg in $20 \mathrm{~mL}$ of water). ${ }^{36}$

\section{Multiple adjuvant instillations}

Patients with low grade Ta disease will benefit from induction chemotherapy followed by maintenance therapy (Fig. 1). There are no published trials that directly compared induction course of MMC to MMC induction with maintenance therapy; however a current meta-analysis suggests that long-term maintenance therapy enhances the effectiveness of MMC induction in preventing recurrences. ${ }^{16}$ Two meta-analyses assessing the impact of intravesical chemotherapy in primary and recurrent NMIBC demonstrated reduction in recurrences with the use of chemotherapy; the benefit appeared to be more significant when at least 1 to 2 years of maintenance therapy was used. ${ }^{37,38}$ Optimal maintenance dose, schedule and duration remain unclear. Importantly, none of the studies incorporated the "optimized" administration of MMC which has been shown to significantly influence the drug's efficacy in a phase III trial. Furthermore, none of the trials directly compared MMC maintenance therapy to a single, immediate postoperative instillation. Patients with low-risk disease who develop recurrent low-grade Ta lesions may receive an induction fol- 
lowed by maintenance course of intravesical chemotherapy (Grade $\mathrm{C}$ recommendation). Meta-analysis of 22 randomized, prospective studies evaluating the role of intravesical chemotherapy for NMIBC did not show any benefit in the reduction of progression rates compared to TURBT alone (Level of evidence 1). ${ }^{39}$ Intravesical gemcitabine and docetaxel have been studied, but there is insufficient evidence to support its superiority over the currently used intravesical chemotherapeutic agents.

\section{Immunotherapy}

\section{$B C G$}

The US Food and Drug Administration approved BCG for the treatment of CIS of the bladder in 1990. Since then, BCG immunotherapy has emerged as the standard against which all new therapies are compared. Six controlled trials carried out from 1985 to 1996 showed that BCG decreases recurrence rates from $67 \%$ to $29 \% .{ }^{40-46}$ Six meta-analyses compared BCG with intravesical chemotherapy; all of them except one ${ }^{47}$ showed superiority of BCG over chemotherapy in terms of decreasing recurrence. ${ }^{47-52}$ BCG following TUR is superior to TUR alone or TUR plus intravesical chemotherapy in decreasing recurrences, particularly in patients with high-risk disease (Level of evidence 1).

BCG is the only intravesical agent that has been shown to affect tumour progression in several randomized trials (level 1). ${ }^{42,43,53}$ Herr and colleagues evaluated 86 patients with high-risk superficial cancer and showed that the disease progression and mortality rates in patients treated with BCG decreased from $35 \%$ to $28 \%$ and $32 \%$ to $14 \%$, respectively. ${ }^{53}$ This apparent advantage is less significant over long-term follow-up at 15 years. ${ }^{54}$ Among several series, BCG induces a complete response rate to CIS in over $70 \% .^{55}$ In a recent meta-analysis involving 24 randomized trials on 4863 patients treated with TUR plus intravesical BCG, TUR alone or TUR plus treatment other than BCG, Sylvester and colleagues demonstrated a statistically significant decrease in progression rates ( $27 \%$ reduction) for patients who received BCG compared with the control group $(9.8 \%$ vs. $13.8 \%$, respectively). ${ }^{48} \mathrm{~A}$ subset analysis demonstrated that the reduction in progression rate was significant only when BCG maintenance was administered. In 2005, Sylvester and colleagues reported their analysis on 12 different randomized trials that included patients with CIS. They compared BCG with different intravesical chemotherapy regimens. ${ }^{56}$ They found a $68 \%$ complete response rate with BCG versus a $48 \%$ complete response rate with chemotherapy. The overall disease-free rates over a median follow up of 3.75 years were $51 \%$ versus $27 \%$ for BCG versus chemotherapy respectively. Similarly, another meta-analyses of 9 randomized trials showed similar results. ${ }^{52}$ Takenaka and colleagues found that the overall response rate to BCG in patients with primary, concomitant or secondary $\mathrm{CIS}$ was $86.6 \%$, with a 5 -year progression-free survival rate of $78.5 \% .{ }^{57}$ Most recurrences or progression occur within the first 5 years. ${ }^{58}$ BCG is the standard of care following TUR for high-risk NMIBC (Grade A recommendation). Patients with intermediate risk NMIBC are recommended to receive either intravesical induction course with chemotherapy or BCG followed by maintenance (Grade B recommendation). Patients with NMIBC who fail intravesical chemotherapy may benefit from BCG induction and maintenance (Grade B recommendation).

\section{Treatment schedule}

BCG is given after 2 to 4 weeks following TURBT to avoid systemic side effects. Treatment schedules have not been established, but there is a universal agreement that only 6 weekly inductions are not enough. ${ }^{59}$ A second induction has been shown to have an additional benefit of about $25 \%$ when used for prophylaxis and 30\% when used for CIS (Level of evidence 3). ${ }^{59,60}$ However, there is sufficient evidence that BCG maintenance, in addition to induction confer reductions in both recurrence and progression (Level of evidence 1). Lamm and colleagues randomized patients with intermediate- and high-risk NMIBC to receive 6 weekly inductions with BCG versus 6 weekly inductions followed by maintenance ( 3 weekly cycles at 3 months and 6 months, then every 6 months up to 36 months). ${ }^{61}$ Patients receiving maintenance were compared with those who did not show improved median recurrence-free and progression-free survival. In a meta-analysis of 24 trials with 4863 patients, Sylvester and colleagues showed a proven superiority of BCG over intravesical chemotherapy. ${ }^{48}$ Progression-free survival was improved only in the patients that received maintenance BCG. Similarly, Bohle and colleagues had similar conclusions in their meta-analysis of 9 trials where 1328 patients with NMIBC treated with adjuvant MMC were compared with 1421 patients treated with adjuvant BCG. ${ }^{49,51}$ With a median follow-up of 26 months, recurrence rates were $46.4 \%$ for patients treated with adjuvant MMC versus $38.6 \%$ for those treated with adjuvant BCG; progression rates were $9.4 \%$ for patients treated with adjuvant MMC versus $7.7 \%$ for those treated with adjuvant BCG ( $p=0.08$, Odds ratio $=0.77)$. When only trials using maintenance were included ( 5 trials), the difference was significant $(p=0.02$, Odds ratio $=0.66)$. The authors concluded that at least 1 year of maintenance BCG was required to show superiority of BCG over chemotherapy in decreasing recurrence or progression. The optimal BCG maintenance schedule has not been determined. We recommend that all patients planning to undergo BCG therapy receive a 6-week induction course followed by the Lamm maintenance protocol, if tolerated (Grade B recommendation). Several European 
studies have demonstrated that BCG can be reduced to $1 / 3$ to $1 / 4$ with a reduction in toxicity but comparable efficacy. ${ }^{44,62,63}$ However, Morales and colleagues have shown that dose reduction is associated with decreased efficacy in North American patients; they hypothesize that a lower immune response may be induced in patients who do not have previous exposure or inoculation with tuberculosis. As such, we recommend a full dose of BCG be given to patients who can tolerate intravesical therapy, particularly in high-risk NMIBC, with dose reduction reserved for tolerance issues (Grade B recommendation).

\section{BCG failure}

BCG failure is defined as the presence of high-grade NMIBC at 6 months from time of TURBT (or at 3 months if the initial tumour is $\mathrm{T} 1 \mathrm{G} 3 / \mathrm{T} 1 \mathrm{HG}$ ) or any worsening of the disease (higher grade, stage or number of recurrences, or appearance of CIS) while on BCG therapy despite initial response to BCG (Level of evidence 2). ${ }^{64}$ In patients with NMIBC treated with an induction course of BCG (without maintenance) and then later developed recurrence of disease, a second induction course may achieve up to $30 \%$ to $50 \%$ response rates. ${ }^{60,65}$ Beyond 2 induction courses with BCG is not recommended as there is a $7 \%$ actuarial risk of progression with each additional course. ${ }^{66}$ Patients with high-risk NMIBC who fail BCG, the option of radical cystectomy should be recommended and discussed with the patient (Grade B recommendation). Herr and colleagues compared the outcome of patients with NMIBC who received a radical cystectomy due to recurrence of disease within 2 years from initial BCG therapy with patients who received radical surgery after 2 years; early radical cystectomy was associated with significantly improved survival in patients with non-muscle invasive recurrence as well as muscle-invasive recurrrence. ${ }^{67}$ After BCG failure, second-line intravesical therapy with combined low-dose BCG and interferon alpha $2 b$ (induction followed maintenance therapy) is a viable option with lower toxicity but may be associated with significant oncologic risk (Level of evidence 3). In a recent large multicentre phase II trial, 467 BCG failure patients receiving low-dose BCG and interferon alpha $2 \mathrm{~b}$ in parallel with 536 BCG naïve patients receiving standard dose BCG with interferon alpha $2 \mathrm{~b} .{ }^{68}$ After a median follow-up of 24 months, $45 \%$ of the BCG failure patients and 59\% of BCG naïve patients were disease-free. ${ }^{69}$ Immediate radical cystectomy may be offered upfront in patients with T1G3/T1HG tumours, highgrade tumours with concomitant $\mathrm{CIS}$, or multiple recurrent high-grade tumours (Grade $\mathrm{C}$ recommendation).

\section{Device-assisted therapy}

Several studies have evaluated the efficacy of device-assisted therapy in the treatment of patients with NMIBC. Two stud- ies have demonstrated that patients with intermediate/highrisk NMIBC treated with MMC combined with hyperthermia (thermochemotherapy) had significantly prolonged recurrence-free survival compared to those treated with MMC alone. $^{22,70}$ Another phase III study demonstrated improved recurrence and progression rates in 108 patients with T1 disease treated with sequential BCG and MMC/electromotive drug administration compared with BCG alone. ${ }^{71}$ Until more studies are completed to further evaluate device-assisted therapies as first- and second-line treatment, no recommendations are possible at the present time.

*Division of Urology, McGill University, Montréal, QC; ${ }^{\dagger}$ University of Texas, MD Anderson Cancer Center, Houston, TX; ҒDepartment of Surgery, University of Toronto, Toronto, ON; §Memorial Sloan Kettering Cancer Center, New York, NY; ${ }^{ \pm}$Division of Urology, University of Alberta, Edmonton, $A B ;{ }^{* *} U B C$, Vancouver, BC; ${ }^{\dagger}$ Faculty of Medicine, University of Western Ontario, London, ON; 㧊Department of Urology, Dalhousie University, Halifax,NS; $§ \S$ Department of Surgery, Staff Urologist, CHUQ-Hôtel-Dieu de Québec, Québec, QC

\section{Competing interests: None declared.}

This paper has been peer-reviewed.

\section{References}

1. Kirkali Z, Chan T, Manoharan M, et al. Bladder cancer: epidemiology, staging and grading, and diagnosis. Urology 2005;66:4-34

2. Chow WH, Lindblad $P$, Gridley $G$, et al. Risk of urinary tract cancers following kidney or ureter stones. J Natl Cancer Inst 1997;89:1453-7.

3. Kantor AF, Hartge P, Hoover RN, et al. Urinary tract infection and risk of bladder cancer. Am J Epidemiol 1984; 119:510-5.

4. Fioriti D, Pietropaolo V, Dal Forno S, et al. Urothelial bladder carcinoma and viral infections: different association with human polyomaviruses and papillomaviruses. Int I Immunopathol Pharmacol 2003;16:283-8.

5. Mostafa MH, Sheweita SA, O'Connor PJ. Relationship between schistosomiasis and bladder cancer. Clin Microbiol Rev 1999;12:97-111.

6. Cannon J, Linke CA, Cos LR. Cyclophosphamide-associated carcinoma of urothelium: modalities for prevention. Urology 1991;38:413-6.

7. Kaldor JM, Day NE, Kittelmann B, et al. Bladder tumours following chemotherapy and radiotherapy for ovarian cancer: a case-control study. Int J Cancer 1995;63:1-6.

8. Clayson DB. Specific aromatic amines as occupational bladder carcinogens. Natl Cancer Inst Monogr 1981;(58):15-9.

9. Droller MJ. Alterations of the p53 gene in occupational bladder cancer in workers exposed to aromatic amines. J Urol 1998;160:618.

10. Piper JM, Tonascia J, Matanoski GM. Heavy phenacetin use and bladder cancer in women aged 20 to 49 years. N Engl J Med 1985;313:292-5.

11. Heney NM. Natural history of superficial bladder cancer. Prognostic features and long-term disease course. Urol Clin North Am 1992;19:429-33.

12. Botteman MF, Pashos CL, Redaelli A, et al. The health economics of bladder cancer: a comprehensive review of the published literature. Pharmacoeconomics 2003;21:1315-30.

13. Avritscher EB, Cooksley CD, Grossman HB, et al. Clinical model of lifetime cost of treating bladder cancer and associated complications. Urology 2006;68:549-53.

14. Babjuk M, Oosterlinck W, Sylvester R, et al. EAU Guidelines on Non-Muscle-Invasive Urothelial Carcinoma of the Bladder. Eur Urol 2008;54:303-14.

15. Montie JE, Abrahams NA, Bahnson RR, et al. Bladder cancer. Clinical guidelines in oncology. J Natl Compr Canc Netw 2006;4:984-1014.

16. Hall MC, Chang SS, Dalbagni $G$, et al. Guideline for the management of nonmuscle invasive bladder cancer (stages Ta, Tl, and Tis): 2007 update. J Urol 2007;178:2314-30. 
17. Zlotta AR, van Vooren JP, Huygen $K$, et al. What is the optimal regimen for BCG intravesical therapy? Are six weekly instillations necessary? Eur Urol 2000;37:470-7.

18. Soloway MS. Overview of treatment of superficial bladder cancer. Urology 1985;26:18-26.

19. Holmang $\mathrm{S}$, Hedelin $\mathrm{H}$, Anderstrom C, et al. The relationship among multiple recurrences, progression and prognosis of patients with stages Ta and $\mathrm{Tl}$ transitional cell cancer of the bladder followed for at least 20 years. J Urol 1995;153:1823-6.

20. Millan-Rodriguez F, Chechile-Toniolo G, Salvador-Bayarri J, et al. Multivariate analysis of the prognostic factors of primary superficial bladder cancer. J Urol 2000;163:73-8.

21, Flamm J, Havelec L. Factors affecting survival in primary superficial bladder cancer. Eur Urol 1990;17:113-8.

22. van der Heijden $A G$, Kiemeney LA, Gofrit $\mathrm{ON}$, et al. Preliminary European results of local microwave hyperthermia and chemotherapy treatment in intermediate or high risk superficial transitional cell carcinoma of the bladder. Eur Urol 2004;46:65-71.

23. Lutzeyer W, Rubben H, Dahm H. Prognostic parameters in superficial bladder cancer: an analysis of 315 cases. J Urol 1982;127:250-2.

24. Herr HW. Restaging transurethral resection of high risk superficial bladder cancer improves the initial response to bacillus Calmette-Guerin therapy. J Urol 2005;174:2134-7.

25. Filbeck $T$, Pichlmeier $U$, Knuechel $R$, et al. Clinically relevant improvement of recurrence-free survival with 5-aminolevulinic acid induced fluorescence diagnosis in patients with superficial bladder tumours. $J$ Urol 2002;168:67-71.

26. Daniltchenko DI, Riedl CR, Sachs MD, et al. Long-term benefit of 5-aminolevulinic acid fluorescence assisted transurethral resection of superficial bladder cancer: 5 -year results of a prospective randomized study. J Urol 2005; 174:2129-33.

27. Herr HW. The value of a second transurethral resection in evaluating patients with bladder tumours. $J$ Urol 1999;162:74-6.

28. Schips L, Augustin H, Zigeuner RE, et al. Is repeated transurethral resection justified in patients with newly diagnosed superficial bladder cancer? Urology 2002;59:220-3.

29. Grimm MO, Steinhoff C, Simon $X$, et al. Effect of routine repeat transurethral resection for superficial bladder cancer: a long-term observational study. J Urol 2003;170:433-7.

30. Fitzpatrick JM, West AB, Butler MR, et al. Superficial bladder tumours (stage pTa, grades 1 and 2): the importance of recurrence pattern following initial resection. J Urol 1986;135:920-2.

31. Sylvester RJ, Oosterlinck W, van der Meijden AP. A single immediate postoperative instillation of chemotherapy decreases the risk of recurrence in patients with stage Ta TI bladder cancer: a meta-analysis of published results of randomized clinical trials. J Urol 2004;171:2186-90.

32. Kaasinen $E$, Rintala $E$, Hellstrom $P$, et al. Factors explaining recurrence in patients undergoing chemoimmunotherapy regimens for frequently recurring superficial bladder carcinoma. Eur Urol 2002;42:167-74.

33. Nieuwenhuijzen JA, Bex A, Horenblas $\mathrm{S}$. Unusual complication after immediate postoperative intravesical mitomycin C instillation. Eur Urol 2003;43:711-2.

34. Oddens JR, van der Meijden AP, Sylvester R. One immediate postoperative instillation of chemotherapy in low risk Ta, T1 bladder cancer patients. Is it always safe? Eur Urol 2004;46:336-8.

35. Racioppi $M$, Porreca A, Foschi N, et al. Bladder perforation: a potential risk of early endovesical chemotherapy with mitomycin C. Urol Int 2005;75:373-5.

36. Au JL, Badalament RA, Wienties MG, et al. Methods to improve efficacy of intravesical mitomycin C: results of a randomized phase III trial. J Natt Cancer Inst 2001;93:597-604.

37. Huncharek $M$, Geschwind JF, Witherspoon $B$, et al. Intravesical chemotherapy prophylaxis in primary superficial bladder cancer: a meta-analysis of 3703 patients from 11 randomized trials. J Clin Epidemiol 2000;53:676-80.

38. Huncharek M, McGarry R, Kupelnick B. Impact of intravesical chemotherapy on recurrence rate of recurrent superficial transitional cell carcinoma of the bladder: results of a meta-analysis. Anticancer Res 2001;21:765-9.

39. Lamm DL, Riggs DR, Traynelis $\mathrm{CL}$, et al. Apparent failure of current intravesical chemotherapy prophylaxis to influence the long-term course of superficial transitional cell carcinoma of the bladder. J Urol 1995:153:1444-50.

40. Lamm DL. Bacillus Calmette-Guerin immunotherapy for bladder cancer. J Urol 1985;134:40-7.

41. Nseyo UO, Lamm DL. Immunotherapy of bladder cancer. Semin Surg Oncol 1997;13:342-9.

42. Herr HW, Pinsky CM, Whitmore WF Jr, et al. Experience with intravesical bacillus Calmette-Guerin therapy of superficial bladder tumours. Urology 1985;25:119-23.

43. Herr HW. Transurethral resection and intravesical therapy of superficial bladder tumours. Urol Clin North Am 1991;18:525-8

44. Pagano F, Bassi P, Milani C, et al. A low dose bacillus Calmette-Guerin regimen in superficial bladder cancer therapy: is it effective? J Urol 1991;146:32-5.

45. Melekos MD, Chionis H, Pantazakos A, et al. Intravesical bacillus Calmette-Guerin immunoprophylaxis of superficial bladder cancer: results of a controlled prospective trial with modified treatment schedule. J Urol 1993; 149:744-8
46. Krege $S$, Giani $G$, Meyer $R$, et al. A randomized multicenter trial of adjuvant therapy in superficial bladder cancer: transurethral resection only versus transurethral resection plus mitomycin C versus transurethral resection plus bacillus Calmette-Guerin. Participating Clinics. J Urol 1996;156:962-6.

47. Huncharek M, Kupelnick B. Impact of intravesical chemotherapy versus BCG immunotherapy on recurrence of superficial transitional cell carcinoma of the bladder: metaanalytic reevaluation. Am J Clin Oncol 2003:26:402-7.

48. Sylvester RJ, van der Meiiden AP, Lamm DL. Intravesical bacillus Calmette-Guerin reduces the risk of progression in patients with superficial bladder cancer: a meta-analysis of the published results of randomized clinical trials. J Urol 2002;168:1964-70.

49. Bohle A, Jocham D, Bock PR. Intravesical bacillus Calmette-Guerin versus mitomycin C for superficial bladder cancer: a formal meta-analysis of comparative studies on recurrence and toxicity. J Urol 2003;169:90-5.

50. Shelley MD, Wilt TJ, Court J, et al. Intravesical bacillus Calmette-Guerin is superior to mitomycin C in reducing tumour recurrence in high-risk superficial bladder cancer: a meta-analysis of randomized trials. BJU Int 2004;93:485-90.

51. Bohle A, Bock PR. Intravesical bacille Calmette-Guerin versus mitomycin C in superficial bladder cancer: formal meta-analysis of comparative studies on tumour progression. Urology 2004;63:682-6.

52. Sylvester RJ, van der Meiiden AP, Witjes JA, et al. Bacillus calmette-guerin versus chemotherapy for the intravesical treatment of patients with carcinoma in situ of the bladder: a meta-analysis of the published results of randomized clinical trials. J Urol 2005;174:86-91.

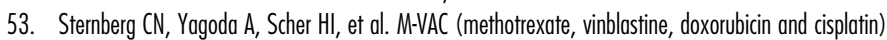
for advanced transitional cell carcinoma of the urothelium. J Urol 1988;139:461-9.

54. Cookson MS, Herr HW, Zhang ZF, et al. The treated natural history of high risk superficial bladder cancer: 15-year outcome. J Urol 1997; 158:62-7.

55. Lamm DL. Carcinoma in situ. Urol Clin North Am 1992;19:499-508.

56. Sylvester RJ, van der Meijden AP, Wities JA, et al. High-grade Ta urothelial carcinoma and carcinoma in situ of the bladder. Urology 2005;66:90-107.

57. Takenaka A, Yamada Y, Miyake H, et al. Clinical outcomes of bacillus Calmette-Guerin instillation therapy for carcinoma in situ of urinary bladder. Int J Urol 2008;15:309-13.

58. Herr HW, Wartinger DD, Fair WR, et al. Bacillus Calmette-Guerin therapy for superficial bladder cancer: a 10-year followup. J Urol 1992; 147:1020-3.

59. Kavoussi LR, Torrence RJ, Gillen DP, et al. Results of 6 weekly intravesical bacillus Calmette-Guerin instillations on the treatment of superficial bladder tumours. J Urol 1988;139: 935-40.

60. Bretton PR, Herr HW, Kimmel M, et al. The response of patients with superficial bladder cancer to a second course of intravesical bacillus Calmette-Guerin. J Urol 1990;143:710-2

61. Lamm DL, Blumenstein BA, Crissman JD, et al. Maintenance bacillus Calmette-Guerin immunotherapy for recurrent TA, Tl and carcinoma in situ transitional cell carcinoma of the bladder: a randomized Southwest Oncology Group Study. J Urol 2000;163:1124-9.

62. Losa A, Hurle R, Lembo A. Low dose bacillus Calmette-Guerin for carcinoma in situ of the bladder: longterm results. J Urol 2000;163:68-71.

63. Mack D, Frick J. Five-year results of a phase II study with low-dose bacille Calmette-Guerin therapy in high-risk superficial bladder cancer. Urology 1995;45:958-61.

64. Herr HW, Dalbagni G. Defining bacillus Calmette-Guerin refractory superficial bladder tumours. J Urol 2003; 169:1706-8.

65. Bui TT, Schellhammer PF. Additional bacillus Calmette-Guerin therapy for recurrent transitional cell carcinoma after an initial complete response. Urology 1997;49:687-90

66. Catalona WJ, Hudson MA, Gillen DP, et al. Risks and benefits of repeated courses of intravesical bacillus Calmette-Guerin therapy for superficial bladder cancer. J Urol 1987;137:220-4.

67. Herr HW, Sogani PC. Does early cystectomy improve the survival of patients with high risk superficial bladder tumours? J Urol 2001;166:1296-9.

68. Joudi FN, Smith BJ, O'Donnell MA. Final results from a national multicenter phase II trial of combination bacillus Calmette-Guerin plus interferon alpha-2B for reducing recurrence of superficial bladder cancer. Urol Oncol 2006;24:344-8.

69. O'Donnell MA, Lilli K, Leopold C. Interim results from a national multicenter phase II trial of combination bacillus Calmette-Guerin plus interferon alfa-2b for superficial bladder cancer. J Urol 2004;172:888-93.

70. Colombo R, Da Pozzo LF, Salonia A, et al. Multicentric study comparing intravesical chemotherapy alone and with local microwave hyperthermia for prophylaxis of recurrence of superficial transitional cell carcinoma. J Clin Oncol 2003;21:4270-6.

71. Di Stasi SM, Giannantoni A, Giurioli A, et al. Sequential BCG and electromotive mitomycin versus BCG alone for high-risk superficial bladder cancer: a randomised controlled trial. Lancet Oncol 2006;7:43-51.

Correspondence: Dr. Wassim Kassouf, Division of Urology, McGill University Health Centre, 1650 Cedar Ave., Rm L8-315, Montréal, QC H3G 1A4; fax: 514-934-8297; wassim.kassoư@muhc.mcgill.ca 